\title{
Exact solutions of a new elliptic Calogero-Sutherland model
}

\author{
D. Gómez-Ullate, A. González-López*, M.A. Rodríguez \\ Departamento de Física Teórica II, Facultad de Ciencias Físicas, Universidad Complutense, 28040 Madrid, Spain
}

Received 21 February 2001; received in revised form 18 April 2001; accepted 3 May 2001

Editor: R. Gatto

\begin{abstract}
A quantum Hamiltonian describing $N$ particles on a line interacting pairwise via an elliptic function potential in the presence of an external field is introduced. For a discrete set of values of the strength of the external potential, it is shown that a finite number of eigenfunctions and eigenvalues of the model can be exactly computed in an algebraic way. @ 2001 Elsevier Science B.V. All rights reserved.
\end{abstract}

PACS: 03.65.Fd; 71.10.Pm; 11.10.Lm

It is well known that the class of exactly solvable problems does not include most physical problems. The development of computer science in the last decades has made possible the use of numerical methods to approximate exact solutions in a wide variety of situations. Yet, the study of exactly solvable models still deserves attention, not only because the knowledge of exact solutions can be used to test approximate methods, but also in its own right, due to the simplicity and mathematical beauty of the models, and the wide range of connections with other fields of physical and mathematical research.

This is illustrated by the renewed interest in the Calogero-Sutherland (CS) models of interacting particles in one dimension, which have been recently applied to many different fields such us quantum spin chains with long range interaction [1], random matrix theory [2], fractional statistics and anyons [3], Yang-Mills theories [4], quantum Hall liquids [5], soliton theory [6], and black holes [7].

The first example of a non-trivial integrable quantum many-body problem was found by Calogero [8], and consists of a system of identical nonrelativistic particles interacting pairwise through an inverse-square potential $v(r)=r^{-2}$, so that the Hamiltonian is

$$
H_{N}=-\sum_{k=1}^{N} \partial_{x_{k}}^{2}+g \sum_{\substack{j, k=1 \\ j \neq k}}^{N} v\left(x_{j}-x_{k}\right) .
$$

\footnotetext{
* Corresponding author. Phone: +34913944596 . Fax: +34913944557.

E-mail addresses: dgu@eucmos.sim.ucm.es (D. Gómez-Ullate), artemio@eucmos.sim.ucm.es (A. González-López), rodrigue@eucmos.sim.ucm.es (M.A. Rodríguez).
} 
By integrable we mean here that a complete commuting set of constants of motion can be explicitly constructed. Soon afterwards, Sutherland [9] established the integrability of the model (1) with an inverse sine-square interaction $v(r)=\sin ^{-2}(r)$.

The most general interaction potential $v$ for which the Hamiltonian (1) is known to be integrable is the Weierstrass $\wp$ function, which includes the rational and trigonometric cases as special limits. The integrability of this potential was proved in the classical case by Calogero and Perelomov by means of a Lax pair representation [10], and its explicit integration was performed by Krichever [11]. Olshanetsky and Perelomov [12] later showed that all these models have an underlying algebraic structure based on root systems of $A_{N}$ algebras, and that integrable models associated to other root systems also exist. In the models treated in Ref. [12] the integrals of motion are related to the radial parts of the Laplace-Beltrami operator on a symmetric space associated to the given root system. These integrable models are obtained from the projection of free motion on a higher-dimensional manifold.

However, integrable Hamiltonians are not necessarily exactly solvable, i.e., one might not be able to find explicitly their spectrum and eigenfunctions. The models with inverse-square and inverse sine-square interaction are known to be exactly solvable, and much literature has in fact been devoted to the study of their eigenfunctions [13], but the more general model with the Weierstrass $\wp$ potential is considerably more difficult to handle. In fact, very few explicit solutions are known in this case, and only for a low number of particles [14] (see also the recent paper [15] for a promising new approach based on second quantization).

The exact solvability of the Calogero-Sutherland models related to classical root systems was established in Refs. [16] and [17] by noting that the Hamiltonian can be mapped by a suitable gauge rotation to an element of the enveloping algebra of a certain realization of $\mathfrak{s l}(N+1)$ admitting finite-dimensional representations (cf. Eq. (11) below). A generalization of this idea was used in Refs. [18] and [19] to construct deformations of the original Calogero model for which only a subset of the spectrum can be exactly computed. Following Refs. [20] and [21], we shall call such models quasi-exactly solvable (QES). A somewhat different approach was followed by Hou and Shifman [22], who obtained a deformation of the $B_{N}$ Calogero model by studying the motions of the zeros of suitable solutions of the time-dependent Schrödinger equation of a well-known QES one-particle polynomial potential. The quasi-exact solvability of the $N$-particle model thus constructed was again proved by mapping its Hamiltonian to a polynomial in the generators of the $\mathfrak{s l}(N+1)$ algebra (11) by a suitable change of variables and gauge transformation. This method was further developed and systematically applied to other families of QES onedimensional one-particle potentials by the authors, thereby obtaining several exactly and quasi-exactly solvable rational and hyperbolic Calogero-Sutherland models [23].

In this Letter we shall present a model of $N$ particles on a line with elliptic pairwise interaction in an external field for which afinite number of eigenfunctions and their corresponding energies can be computed algebraically. Since the discrete spectrum is easily seen to be infinite (the potential diverges on the boundary of a compact region), the model is quasi-exactly solvable. We shall only sketch here the main ideas behind the proof of these results, referring the reader to Ref. [23] for a complete description of the method used.

Consider the $N$-body quantum Hamiltonian

$$
H_{N}=-\sum_{k=1}^{N} \partial_{x_{k}}^{2}+V_{N}(\mathbf{x})
$$

with potential

$$
V_{N}(\mathbf{x})=c_{m} \sum_{k=1}^{N} \wp\left(x_{k}+\mathrm{i} \beta\right)+4 b(b-1) \sum_{k=1}^{N} \wp\left(2 x_{k}\right)+a(a-1) \sum_{\substack{j, k=1 \\ j \neq k}}^{N}\left[\wp\left(x_{j}-x_{k}\right)+\wp\left(x_{j}+x_{k}\right)\right]
$$


where $a$ and $b$ are positive real parameters, $m$ is a non-negative integer,

$$
c_{m}=2(2 b+m+a(N-1))(2 b+2 m+2 a(N-1)+1)>0,
$$

and $\wp(z) \equiv \wp\left(z \mid g_{2}, g_{3}\right)$ denotes the Weierstrass $\wp$ function with invariants $g_{2}, g_{3} \in \mathbf{R}$. If $g_{2}$ and $g_{3}$ satisfy the inequality $g_{2}^{3}>27 g_{3}^{2}$, then $\wp(z)$ has two fundamental periods $2 \alpha$ and $2 \mathrm{i} \beta$ which are real and purely imaginary, respectively [24]. In this case $\wp(x+\mathrm{i} \beta)$ is real and regular (analytic) for all real values of $x$, with real period $2 \alpha$. On the other hand, $\wp(x)$ is real for real $x$ and diverges as $(x-2 n \alpha)^{-2}$ when $x$ tends to an integer multiple $2 n \alpha$ of the real period $2 \alpha$. Thus, the configuration space for the Hamiltonian (2), (3) can be taken as the bounded region of $\mathbf{R}^{N}$

$$
0<x_{N}<x_{N-1}<\cdots<x_{1}<\alpha .
$$

Since the potential (3) is confining in this region, the spectrum of $H_{N}$ is infinite and purely discrete, and the boundary condition defining its eigenfunctions $\psi_{k}(\mathbf{x})$ is their vanishing on the boundary of (5).

The potential (3) with $c_{m}=0$ is of $C_{N}$ type [12]

$$
4 b(b-1) \sum_{k=1}^{N} v\left(2 x_{k}\right)+a(a-1) \sum_{\substack{j, k=1 \\ j \neq k}}^{N}\left[v\left(x_{j}-x_{k}\right)+v\left(x_{j}+x_{k}\right)\right],
$$

with interaction potential $v(r)=\wp(r)$. The term proportional to $c_{m}$ in (3) can be viewed as the contribution of an external field with potential $\wp(r+\mathrm{i} \beta)$.

We shall now show that, when the parameter $m$ is a non-negative integer, one can algebraically compute a finite number — depending on $m$, see (14) below — of eigenvalues and eigenfunctions of the Hamiltonian (2), (3). These algebraic eigenfunctions have the form

$$
\psi_{k}(\mathbf{x})=\mu(\mathbf{x}) \chi_{k}(\mathbf{z}),
$$

where

$$
\mu(\mathbf{x})=\prod_{j<l}\left[\wp\left(x_{j}+\mathrm{i} \beta\right)-\wp\left(x_{l}+\mathrm{i} \beta\right)\right]^{a} \prod_{j}\left[\wp^{\prime}\left(x_{j}+\mathrm{i} \beta\right)\right]^{b},
$$

and $\chi_{k}(\mathbf{z})$ is a suitable completely symmetric polynomial of degree at most $\mathrm{Nm}$ in the variables

$$
z_{j}=\wp\left(x_{j}+\mathrm{i} \beta\right), \quad j=1, \ldots, N .
$$

The exact solutions of the rational and trigonometric $C_{N}$ - in fact, $B C_{N}$ - models also assume the form (6), but in those cases $\mu$ can be factorized over the system of positive roots, i.e., it has the form

$$
\mu=\prod_{j<k}\left[f\left(x_{j}-x_{k}\right) f\left(x_{j}+x_{k}\right)\right]^{a} \prod_{k}\left[f\left(2 x_{k}\right)\right]^{b},
$$

where $f(x)=x$ in the rational case and $f(x)=\sin x$ in the trigonometric case. Moreover, in both cases $\mu$ coincides with the ground-state wave function of the system. By contrast, the function $\mu$ in (7) cannot be factorized over the system of positive roots for any function $f(x)$, and is not the ground-state wave function of the Hamiltonian (2), (3). As a matter of fact, it was shown in [25] that the most general potential allowing for the factorization (9) does not include the elliptic case. These are some of the reasons why it has been so difficult to obtain explicit solutions of the elliptic CS models, in contrast with their rational and trigonometric counterparts.

When the parameters $a$ and $b$ are positive, the functions (6) are regular in the region (5), and they automatically vanish on its boundary on account of the identities $\wp^{\prime}(\mathrm{i} \beta)=\wp^{\prime}(\alpha+\mathrm{i} \beta)=0$ (see Ref. [24]). Thus, to show that $\psi_{k}$ in Eq. (6) is an eigenfunction of $H_{N}$ we only have to check that it satisfies the Schrödinger equation $\left(H_{N}-E_{k}\right) \psi_{k}=0$ 
in the open region (5). Equivalently, $\chi_{k}$ must be a solution of the equation $\left(\bar{H}_{N}-E_{k}\right) \chi_{k}=0$, where the gauge Hamiltonian $\bar{H}_{N}$ is defined by

$$
\bar{H}_{N}=\mu^{-1} H_{N} \mu \text {. }
$$

Note that, by the standard properties of the Weierstrass function [24], $\mu$ does not vanish in the region (5).

The quasi-exact solvability of the Hamiltonian (3) when $m$ is a non-negative integer can be established as follows. Using the method developed in Ref. [23], it can be shown that $\bar{H}_{N}$ is a quadratic combination of the generators of $\mathfrak{s l}(N+1)$ in the representation

$$
\mathcal{D}_{k}=\partial_{\tau_{k}}, \quad \mathcal{N}_{j k}=\tau_{j} \partial_{\tau_{k}}, \quad \mathcal{U}_{k}=\tau_{k}\left(m-\sum_{i=1}^{N} \tau_{i} \partial_{\tau_{i}}\right) \quad j, k=1, \ldots, N,
$$

where

$$
\tau_{k}=\sum_{1 \leqslant i_{1}<i_{2}<\cdots<i_{k} \leqslant N} z_{i_{1}} z_{i_{2}} \cdots z_{i_{k}}, \quad k=1, \ldots, N,
$$

are the elementary symmetric functions of the variables $z_{k}$ [16]. When $m$ is a non-negative integer, the generators (11) obviously preserve the finite-dimensional polynomial space

$$
\mathcal{M}_{m}=\operatorname{span}\left\{\tau_{1}^{l_{1}} \tau_{2}^{l_{2}} \cdots \tau_{N}^{l_{N}}: l_{i} \in \mathbf{N}, \sum_{i=1}^{N} l_{i} \leqslant m\right\} .
$$

Since the gauge Hamiltonian $\bar{H}_{N}$ is a quadratic polynomial in the operators (11), it also preserves the space (13). It follows that $\bar{H}_{N}$ has (at most)

$$
\operatorname{dim} \mathcal{M}_{m}=\left(\begin{array}{c}
m+N \\
m
\end{array}\right)
$$

eigenfunctions $\chi_{k}$ lying in $\mathcal{M}_{m}$, which can be algebraically computed, along with their corresponding eigenvalues, simply by diagonalizing the finite-dimensional matrix of $\bar{H}_{N} \mid \mathcal{M}_{m}$. The elements of $\mathcal{M}_{m}$, being polynomials in the symmetric variables $\tau_{k}$ of degree at most $m$, are symmetric polynomials in $\mathbf{z}$ of degree not greater than $\mathrm{Nm}$. It follows from Eq. (10) that the physical Hamiltonian (2), (3) possesses (at most) $\operatorname{dim} \mathcal{M}_{m}$ algebraically computable eigenfunctions of the form (6), (7), with $\chi_{k}(\mathbf{z})$ a symmetric polynomial of degree at most $N m$, as claimed.

The algebra $\mathfrak{s l}(N+1)$, which plays a fundamental role in the partial integrability of the Hamiltonian (2), (3), is sometimes called a hidden symmetry algebra [26], since in this approach the Hamiltonian need not be a Casimir element. Note that the Hamiltonian (2), (3) must certainly have eigenfunctions which do not belong to the algebraic sector (6), (7), since its discrete spectrum is infinite. An interesting open problem in this respect would be to analyze the position in the spectrum and the degeneracy of the algebraic eigenvalues. It should also be emphasized that, since the model (2), (3) is quasi-exactly solvable, it is not possible to give a procedure to diagonalize the Hamiltonian for arbitrary values of $m$ and $N$. However, for any specific value of these parameters the Hamiltonian can be diagonalized in the finite-dimensional subspace $\mu \mathcal{M}_{m}$, and in this way a finite subset of its spectrum can be exactly computed.

As a simple example, consider the problem for $N=2$ and $m=2$, for which the potential reads

$$
\begin{aligned}
V_{2}\left(x_{1}, x_{2}\right)= & 2(2 b+a+2)(2 b+2 a+5)\left[\wp\left(x_{1}+\mathrm{i} \beta\right)+\wp\left(x_{2}+\mathrm{i} \beta\right)\right] \\
& +4 b(b-1)\left[\wp\left(2 x_{1}\right)+\wp\left(2 x_{2}\right)\right]+2 a(a-1)\left[\wp\left(x_{1}-x_{2}\right)+\wp\left(x_{1}+x_{2}\right)\right] .
\end{aligned}
$$

Note that this is intrinsically a two-body problem, since the potential (15) is not translation-invariant. The number of algebraic eigenstates is (at most) $\operatorname{dim} \mathcal{M}_{2}=6$, and the matrix of the restriction $\left.\bar{H}_{2}\right|_{\mathcal{M}_{2}}$ with respect to the 
Table 1

Algebraic eigenvalues $E_{i}$ of the Hamiltonian (2), (15) and their numerical approximations

\begin{tabular}{lcc}
\hline & Exact & Numerical \\
\hline$E_{0}$ & -86.5484 & -86.40 \\
$E_{1}$ & -43.2786 & -42.96 \\
$E_{2}$ & -10.0288 & -9.64 \\
$E_{3}$ & 12.5045 & 13.11 \\
$E_{4}$ & 46.2657 & 46.97 \\
$E_{5}$ & 81.0857 & 81.34 \\
\hline
\end{tabular}

canonical basis $\left\{1, \tau_{1}, \tau_{2}, \tau_{1}^{2}, \tau_{1} \tau_{2}, \tau_{2}^{2}\right\}$ of $\mathcal{M}_{2}$ is easily computed, with the result

$$
\left(\begin{array}{cccccc}
0 & g_{2}(2 a+2 b+1) & -2 a g_{3} & 4 g_{3} & 0 & 0 \\
16 a+24 b+20 & 0 & g_{2}(b+1 / 2) & 4 g_{2}(a+b+1) & 2 g_{3}(1-a) & 0 \\
0 & 8 a+24 b+12 & 0 & 0 & g_{2}(2 a+2 b+5) & -4 g_{3}(a+1) \\
0 & 8 a+12 b+14 & 0 & 0 & g_{2}(b+1 / 2) & 2 g_{3} \\
0 & 0 & 8 a+12 b+14 & 16(a+3 b+3) & 0 & g_{2}(2 b+3) \\
0 & 0 & 0 & 0 & 8 a+24 b+28 & 0
\end{array}\right) .
$$

The eigenvalues of this matrix are the algebraic energies of the physical Hamiltonian $\mathrm{H}_{2}$, and its eigenvectors give the components of the corresponding functions $\chi_{k}$ in Eq. (6) with respect to the canonical basis of $\mathcal{M}_{2}$.

Take, for instance, the following generic values of the coupling constants and the invariants of $\wp(x)$ :

$$
a=2, \quad b=3 / 2, \quad g_{2}=3, \quad g_{3}=2 / 3,
$$

for which the half-periods are approximately

$$
\alpha=1.31523, \quad \mathrm{i} \beta=1.61809 \mathrm{i} .
$$

The matrix (16) has six real eigenvalues, so that the Hamiltonian (2), (15) possesses six algebraic energies, which have been listed in Table 1. In this table the (exact) algebraic eigenvalues of $H_{2}$ have been compared with a numerical approximation of the six lowest energy levels obtained by solving the Schrödinger equation in the triangle $0<x_{2}<x_{1}<\alpha$ using a finite element method. The approximate agreement between the two columns of Table 1 shows that the algebraic eigenvalues are in this case the lowest energy states of the system, although there is no guarantee that this should still be true in the general case. The polynomials $\chi_{k}$ corresponding to the six algebraic eigenfunctions of the potential (15), (17), written in terms of the symmetric variables $\tau_{1}=z_{1}+z_{2}$ and $\tau_{2}=z_{1} z_{2}$, are listed in Table 2. The fact that $\psi_{0}=\mu \chi_{0}$ is the ground state of the system is immediately apparent if we note that $\chi_{0}$ can be expressed in terms of the variables $z_{k}$ as

$$
\begin{aligned}
\chi_{0}= & 2.2349\left(z_{2}-0.82835\right)\left(z_{2}-0.54017\right)-9.778 z_{1}\left(z_{2}-0.79769\right)\left(z_{2}-0.39212\right) \\
& +9.0382 z_{1}^{2}\left(z_{2}-0.75385\right)\left(z_{2}-0.32801\right) .
\end{aligned}
$$

For the values of the invariants $g_{2}$ and $g_{3}$ given in (17), we have [24]

$$
e_{3}=-0.72011 \leqslant z_{k}=\wp\left(x_{k}+\mathrm{i} \beta\right) \leqslant e_{2}=-0.240851 .
$$

We thus see from the previous expression that $\chi_{0}$ is positive everywhere. Since, by (9), $\mu$ has no zeros in the open triangle $0<x_{2}<x_{1}<\alpha$, it follows that $\psi_{0}$ does not vanish in this triangle, and is therefore the ground state of the potential (15). 
Table 2

Polynomials $\chi_{k}$ corresponding to the algebraic eigenfunctions $\psi_{k}=\mu \chi_{k}$ of the Hamiltonian (2), (15). In all cases, the coefficient of 1 has been normalized to unity

\begin{tabular}{lrrrrr}
\hline & \multicolumn{1}{c}{$\tau_{1}$} & \multicolumn{1}{c}{$\tau_{2}$} & \multicolumn{1}{c}{$\tau_{1}^{2}$} & \multicolumn{1}{c}{$\tau_{1} \tau_{2}$} & \\
\hline$\chi_{0}$ & -3.05847 & 7.16428 & 2.23486 & -9.77802 & 9.03820 \\
$\chi_{1}$ & -3.30084 & -10.91680 & 2.56123 & 13.46220 & -24.88480 \\
$\chi_{2}$ & 0.40412 & 5.10674 & -2.29117 & -0.77221 & 6.15988 \\
$\chi_{3}$ & -3.92728 & -37.98890 & 2.04575 & 14.73410 & 94.26430 \\
$\chi_{4}$ & 1.64224 & -2.74560 & -0.17611 & -10.47200 & -18.10750 \\
$\chi_{5}$ & 3.89416 & 8.30786 & 3.66754 & 15.10020 & 14.89800 \\
\hline
\end{tabular}

\section{Acknowledgements}

The authors gratefully acknowledge the partial financial support of the DGES under grant PB98-0821. They would also like to thank A.M. Perelomov and O. Ragnisco for several useful conversations.

\section{References}

[1] F.D.M. Haldane, Phys. Rev. Lett. 60 (1988) 635;

B.S. Shastry, Phys. Rev. Lett. 60 (1988) 639;

A.P. Polychronakos, Phys. Rev. Lett. 70 (1993) 2329.

[2] B.D. Simons, P.A. Lee, B.L. Altshuler, Phys. Rev. Lett. 70 (1993) 4122 ;

B.D. Simons, P.A. Lee, B.L. Altshuler, Phys. Rev. Lett. 72 (1994) 64.

[3] A. Polychronakos, Nucl. Phys. B 324 (1989) 597;

Z.N.C. Ha, Phys. Rev. Lett. 73 (1994) 1574;

Z.N.C. Ha, Nucl. Phys. B. 435 [FS] (1995) 604;

S.B. Isakov, G. Lozano, S. Ouvry, Nucl. Phys. B 552 (1999) 677.

[4] J.A. Minahan, A.P. Polychronakos, Phys. Lett. B 326 (1994) 288;

A. Gorskii, N. Nekrasov, Nucl. Phys. B 414 (1994) 213;

E. D'Hoker, D.H. Phong, Nucl. Phys. B 513 (1998) 405.

[5] H. Azuma, S. Iso, Phys. Lett. B 331 (1994) 107;

S. Iso, S.J. Rey, Phys. Lett. B 352 (1995) 111.

[6] A.P. Polychronakos, Phys. Rev. Lett. 74 (1995) 5153.

[7] G.W. Gibbons, P.K. Townsend, Phys. Lett. B 454 (1999) 187.

[8] F. Calogero, J. Math. Phys. 12 (1971) 419.

[9] B. Sutherland, Phys. Rev. A 4 (1971) 2019.

[10] F. Calogero, Lett. Nuovo Cimento 13 (1975) 411;

A.M. Perelomov, Lett. Math. Phys. 1 (1977) 531.

[11] I.M. Krichever, Funct. Anal. Appl. 14 (1980) 282.

[12] M.A. Olshanetsky, A.M. Perelomov, Phys. Rep. 94 (1983) 313.

[13] R. Stanley, Adv. Math. 77 (1988) 76;

L. Lapointe, L. Vinet, Commun. Math. Phys. 178 (1996) 425.

[14] J. Dittrich, V.I. Inozemtsev, J. Phys. A: Math. Gen. 26 (1993) L753;

F. Gavrilov, A.M. Perelomov, J. Math. Phys. 40 (1999) 6339.

[15] E. Langmann, math-ph/0007036, v2;

E. Langmann, math-ph/0102005.

[16] W. Rühl, A.V. Turbiner, Mod. Phys. Lett. A 10 (1995) 2213.

[17] L. Brink, A. Turbiner, N. Wyllard, J. Math. Phys. 39 (1998) 1285.

[18] A. Minzoni, M. Rosenbaum, A. Turbiner, Mod. Phys. Lett. A 11 (1996) 1977.

[19] Y. Brihaye, P. Kosinski, Mod. Phys. Lett. A 14 (1999) 2579. 
[20] A.V. Turbiner, A.G. Ushveridze, Phys. Lett. A 126 (1987) 181.

[21] A.V. Turbiner, Commun. Math. Phys. 118 (1988) 467.

[22] X. Hou, M. Shifman, Int. J. Mod. Phys. A 14 (1999) 2993.

[23] D. Gómez-Ullate, A. González-López, M.A. Rodríguez, J. Phys. A: Math. Gen. 33 (2000) 7305.

[24] E.T. Whittaker, G.N. Watson, A Course in Modern Analysis, 4th edn., Cambridge Univ. Press, 1927.

[25] F. Calogero, Lett. Nuovo Cimento 13 (1975) 507.

[26] M.A. Shifman, Int. J. Mod. Phys. A 4 (1989) 2897. 